\title{
Using Intense Laser on the New Laser Fusion and Calculating Formation Factor of a Cloud and Penetrability in $\mathrm{D}^{+3} \mathrm{He}$ Reaction
}

\author{
S. N. Hoseinimotlagh, S. Sadeghi, and F. Mazafarikoroosh
}

\begin{abstract}
In this paper a new laser fusion by using PW laser is discussed for $\mathrm{D}^{+3}$ He reaction by considering its benefits. The feasibility of a new approach of laser fusion in plasma without implosion has been proposed and is discussed using an intense laser. The cross section of the nuclear reaction is increased by enhancing the penetrability of nuclei through the Coulomb barrier. In this approach, an intense laser field of more than $\mathbf{1 0 0}$ PW was required to distort the Coulomb barrier to obtain enough penetrability .In the present paper we have calculated the penetrability of nuclei and the formation factor by using available relations.
\end{abstract}

Index Terms-Fusion, barrier, laser, penetrability.

\section{INTRODUCTION}

Undoubtedly one of the most important subjects that a peaceful world in 21th century confront will be provide suitable, safe, clean and cheap energy. Energy is necessary for feeding, clothing; housing and protecting the billions of humans live in the earth. One of the energy sources is nuclear fusion [1]. Fusion energy that is the energy source of the stars is caused from the nuclear fusion reactions. The concepts of thermonuclear fusion brought up by Gamow and Teller for the first time [2]. People like Nockles and Livermorr suggested an interesting method of compression and afterward lasers with high yield developed [3]. But in this case one needs ignition and to radiography we need symmetry, uniformity with high accuracy and high ignition power, creates an indirect target which explosion efficiency is not suitable.

Therefore one needs a laser that permits access to a newer method of nuclear reaction induced by laser. The final goal of fusion research is combination of permanent and clean energy in order to get cheap energy. From this point of view one suggests new laser fusion that is consider by a high intensity laser field [4].In this new scheme for enough penetrability in order to Hole - bore, we need a laser with power of more than $100 \mathrm{PW}$ [3]. In this method, ignition is not necessary and we don't require symmetry and uniformity of lasers. Regarding produce clean energy in nuclear reaction $\mathrm{D}+3 \mathrm{He}$ we consider new methods of laser fusion for this reaction and finally calculate penetrability and formation

Manuscript received November 9, 2013; revised January 17, 2014. This work was supported in part by the Department of Physics, Shiraz branch Islamic Azad University, Shiraz, Iran.

The authors are with Department of Physics, Shiraz branch Islamic Azad University, Shiraz, Iran (e-mail: hoseinimotlagh@hotmail.com, sara_sadeghi1368@yahoo.com,e.mozaffari10@yahoo.com). factor of the cloud. Around the center of the nuclei there is a nuclear potential well with a $5 \mathrm{fm}$ radius. But in real cases, there is mesons for nucleon that act as the force of gravity. Intense laser concentrated and is injected into the plasma with the shape of Gaussian beam. Then the laser is propagated throughout the plasma and the intense field is used by the laser along the center of laser direction. This field changes the coulomb barrier in hole- boring. The hole-bored nucleon forms a cloud of Debroli wave probability that expands with velocity of hole- bored nucleon group. This expansion in the oscillating laser field is preserved with uncertainty principle. The hole- bored nucleons form a cloud surround the nucleus and when the hole- bored nuclei collide each other quickly produce a compound nucleus, act as a normal nuclear reaction and this action occurs throughout the laser pulse. After this laser pulse, nuclei are diffused in the clouds and the probability of collisions is decreased and finally the occurrence of reactions is reduced. In this paper we use the $\mathrm{D}^{+3} \mathrm{He}$ fuel. Due to several advantages this reaction has, like reducing radioactive damage, less activating, un-breeding of tritium blankets, high efficiency in direct energy transformation, easier keeping and etc, are favored a lot [4]. One of the other subjects that make this reaction more attractive is lack of producing neutrons in the products. The original assumptions in these new schemes are [5]: 1- plasma is formed with a density more than $\frac{10^{21}}{\mathrm{~cm}^{3}} \cdot 2$ - The wavelength of laser is 1.06 micrometer and therefore solid state laser is a suitable choice for this intense laser in EW output. 3- In penetrability, the nucleon energy level is important; here the energy level $\mathrm{E}$ changes from $+5 \mathrm{keV}$ to 1 $\mathrm{keV}$. 4- The number of cycles corresponds to formation factor of cloud. 5- The lifetime of cloud limited by a pion in the time of $10 \mathrm{~ns}$ in free space.

\section{Theory Governing ON THE Predicted System}

In this scheme coulomb barrier and laser field is defined as [5], [6]:

$$
\begin{aligned}
& \varphi_{t o t}=\frac{z_{l} e}{4 \pi \varepsilon_{0} r}+\varphi_{L}-V_{0} \frac{e^{-\frac{r}{r_{0}}}}{\frac{r}{r_{0}}}= \\
& \frac{z_{l} e}{4 \pi \varepsilon_{0} r}-A \sin (\omega t) r-V_{0} \frac{e^{-\frac{r}{r_{0}}}}{\frac{r}{r_{0}}}
\end{aligned}
$$


In equation (1), the first term is the coulomb field. The second term is also laser field in which [4]:

$$
A(V / m)=2.7 \times 10^{3} I^{\frac{1}{2}}\left(\mathrm{w} / \mathrm{cm}^{2}\right)
$$

In equation (2) is the power density of laser, $\varphi_{L}=-E r$ and $\mathrm{E}=A \sin (\omega t)$. The third term is the nuclear potential with pions and in this scheme we have used Yukawa potential one can write that using $V_{0}=109 \mathrm{MeV}$ where $r$ is distance in of terms; and $r_{0}=1.13 \mathrm{fm}$ in normal situation. Considering two boundary states for this potential in the laser peak [6] we have:

$$
\begin{aligned}
& U_{1}=\left(\frac{z_{1} e}{4 \pi \varepsilon_{0} r}-A r\right) z_{2} e-V_{0} \frac{e^{-\frac{r}{r_{0}}}}{\frac{r}{r_{0}}} \\
& U_{2}=\left(\frac{z_{1} e}{4 \pi \varepsilon_{0} r}+A r\right) z_{2} e-V_{0} \frac{e^{-\frac{r}{r_{0}}}}{\frac{r}{r_{0}}}
\end{aligned}
$$

In $U_{1}$ equation, we have the height of the barrier can be reduced. Here $Z_{1}=1$ and $Z_{2}=2$. The nucleon transition rate [5], [6] that passes throughout the barrier is expressed by:

$$
T=\exp \left(-2 \int_{R}^{R_{I}} \beta(r) d r\right)
$$

In equation (5) we have:

$$
\beta(r)=\left(\frac{2 m}{\hbar^{2}}\right)^{\frac{1}{2}}\left[U_{1}(r)-E\right]^{\frac{1}{2}}
$$

In which $\hbar=6.58217 \times 10^{-22} \mathrm{MeV} . s \quad \mathrm{MeV} . \mathrm{s} \quad$ and $m=938.28 \mathrm{MeV} / \mathrm{c}^{2}$ for proton. $\mathrm{E}$ is the nucleon energy level in range of $0 \mathrm{ev}$ to $10 \mathrm{keV} \mathrm{A}$ simple transition rate $\mathrm{T}$ modifies and penetrability $\mathrm{P}$ is defined [6] as:

$$
P=f T
$$

In equation (5) $\mathrm{f}$ is the collision time of nucleus with interior wall causes from nuclear potential and coulomb barrier. The penetrability value as shows in Fig. 1 and Fig. 2, calculates by using equations (5), (6) and (7) with different parameters. Here we define $\mathrm{F}$ as a formation factor of a holebored nucleon cloud after penetrability. The probability of the cloud formation can be written as $F=p \times N_{c} N_{c}$ is the number of cycles for laser pulses that can be varied from 200 to 2000 . Note that when F approaches to 1 , saturation occurs by discharge nuclei. This effect is obvious for the results from the Fig. 3. In this region for the saturation level [7] we have:

$$
F_{S}=F /(F+1)
$$

\section{NUMERICAL CALCULATIONS}

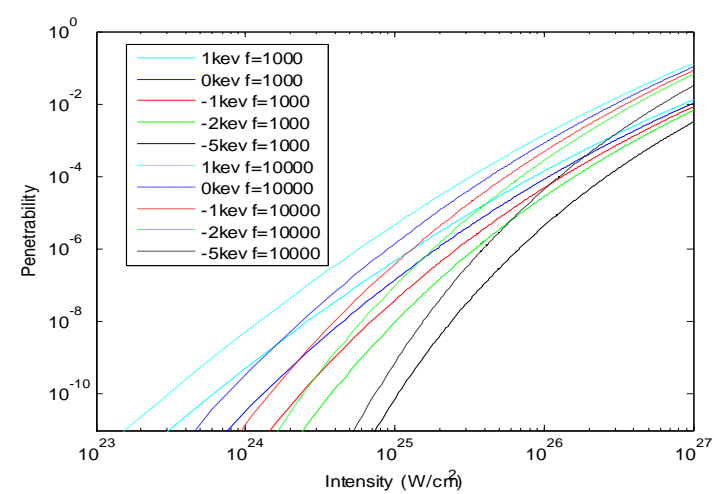

Fig. 1. Calculated penetrability with a power peak of the laser in $\mathrm{D}+{ }^{3} \mathrm{He}$ reaction.

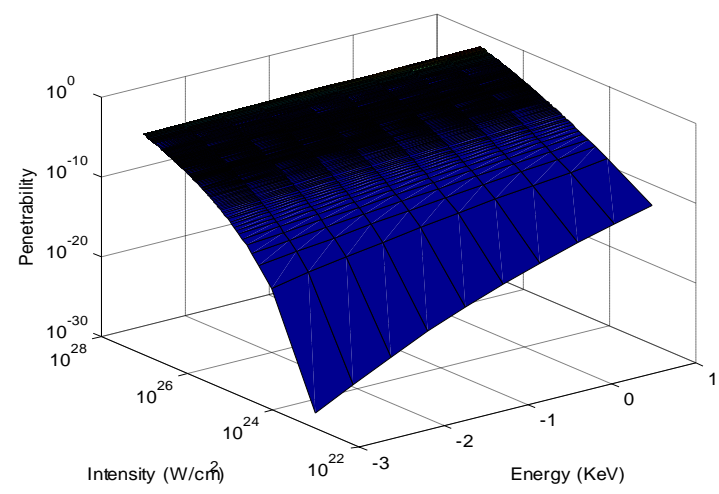

Fig. 2. Three dimensional variation of calculated penetrability versus power peak of the laser and different energies in $\mathrm{D}+{ }^{3} \mathrm{He}$ reaction.

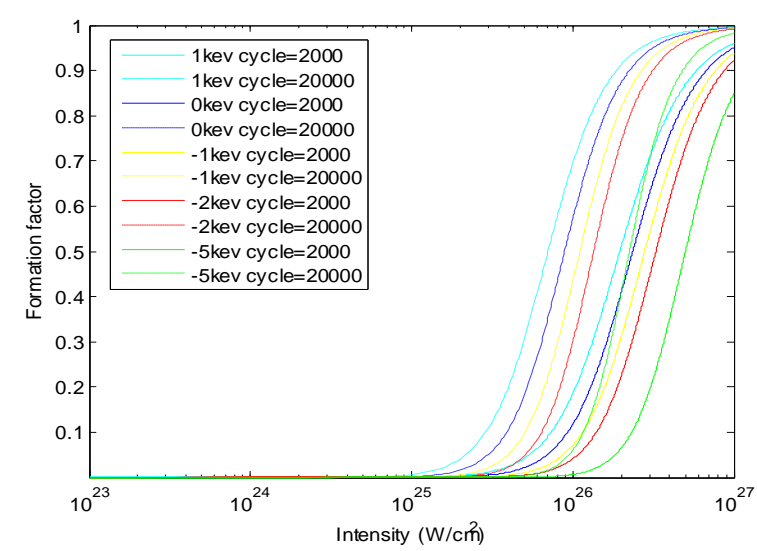

Fig. 3. The 2-D diagram of formation factors of the clouds in cases of long pulses in $\mathrm{D}+{ }^{3} \mathrm{He}$ reaction.

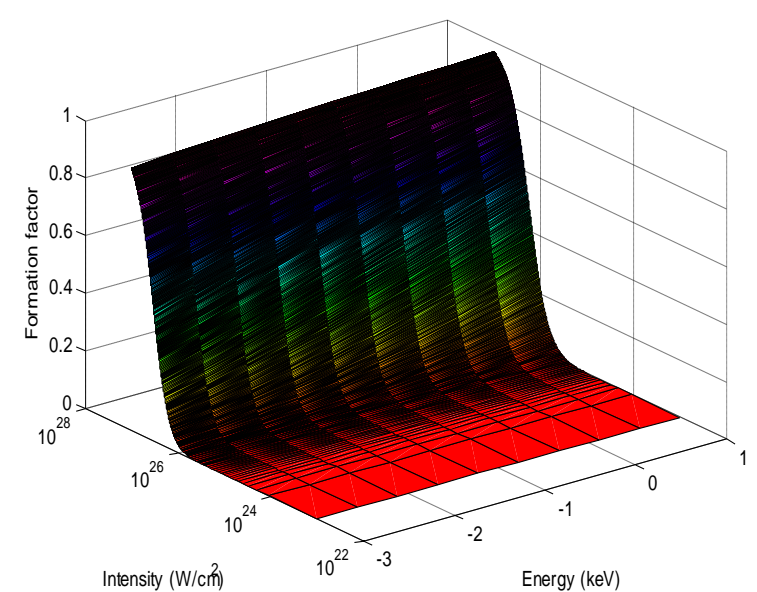

Fig. 4. 3-D diagram of formation factor versus energy and power intensity of laser for $\mathrm{D}+{ }^{3} \mathrm{He}$ reaction. 
The usual results from equation(8) are shown in Fig. 3 and Fig. 4. Events show as 2 cases of number of cycles for 2000 along 900fs pulse and 20000 along 10ps pulse. The calculated values of penetrability for intensity, energy and the collision time of nucleon with interior wall are presented in Table I. Also the calculated values of formation factor of a cloud for intensity the number of cycles of laser pulses, energy and power intensity of laser are presented in Table II.

TABLE I: THE NUMERICAL VALUES OF CALCULATED PENETRABILITY FOR DIFFERENT ENERGY VALUES, POWER INTENSITY OF LASER AND THE COLLISION TIME OF NUCLEON WITH INN

\begin{tabular}{|c|c|c|c|}
\hline penetrability & $\begin{array}{l}\text { intensity } \\
\left(\mathrm{W} / \mathbf{c m}^{2}\right)\end{array}$ & $\begin{array}{c}\text { energy } \\
(\mathrm{keV})\end{array}$ & $\mathbf{f}$ \\
\hline $6.975 \mathrm{e}-010$ & $1.1 \mathrm{e}+024$ & 1 & 1000 \\
\hline 0.0001038 & $8.764 \mathrm{e}+025$ & 1 & 1000 \\
\hline $6.975 \mathrm{e}-009$ & $1.1 \mathrm{e}+024$ & 1 & 10000 \\
\hline 0.001119 & $9.064 \mathrm{e}+025$ & 1 & 10000 \\
\hline $4.253 \mathrm{e}-009$ & $5.602 \mathrm{e}+024$ & -1 & 1000 \\
\hline 0.002622 & $5.598 \mathrm{e}+026$ & -1 & 1000 \\
\hline $5.974 \mathrm{e}-008$ & $6.102 \mathrm{e}+024$ & -1 & 10000 \\
\hline 0.008371 & $3.282 \mathrm{e}+026$ & -1 & 10000 \\
\hline $1.24 \mathrm{e}-011$ & $7.603 e+024$ & -5 & 1000 \\
\hline 0.0002687 & $3.753 e+026$ & -5 & 1000 \\
\hline $1.24 \mathrm{e}-010$ & $7.603 e+024$ & -5 & 10000 \\
\hline 0.0004372 & $2.012 \mathrm{e}+026$ & -5 & 10000 \\
\hline 0.04016 & $8.918 \mathrm{e}+025$ & -5 & 20000 \\
\hline 0.8611 & $4.195 e+026$ & -5 & 20000 \\
\hline
\end{tabular}

TABLE II: NUMERICAL VALUES OF CALCULATED FORMATION FACTOR OF A CLOUD FOR DIFFERENT VALUES OF INTENSITY, ENERGY AND THE NUMBER OF LASER PULSES

\begin{tabular}{|c|c|c|c|}
\hline $\begin{array}{c}\text { Cloud } \\
\text { formation } \\
\text { factor }\end{array}$ & $\begin{array}{c}\text { Intensity } \\
\left(\mathbf{W} / \mathbf{c m}^{2}\right)\end{array}$ & $\begin{array}{c}\text { Energy } \\
(\mathbf{k e V})\end{array}$ & $\begin{array}{c}\text { Number of laser } \\
\text { pulses. }\end{array}$ \\
\hline 0.01124 & $2.913 \mathrm{e}+025$ & 1 & 2000 \\
\hline 0.9128 & $6.367 \mathrm{e}+026$ & 1 & 2000 \\
\hline 0.08637 & $2.712 \mathrm{e}+025$ & 1 & 20000 \\
\hline 0.9932 & $7.608 \mathrm{e}+026$ & 1 & 20000 \\
\hline 0.002018 & $3.813 \mathrm{e}+025$ & -2 & 2000 \\
\hline 0.8949 & $8.519 \mathrm{e}+026$ & -2 & 2000 \\
\hline 0.06108 & $5.415 \mathrm{e}+025$ & -2 & 20000 \\
\hline 0.9708 & $5.506 \mathrm{e}+026$ & -2 & 20000 \\
\hline 0.01272 & $1.212 \mathrm{e}+026$ & -5 & 2000 \\
\hline 0.769 & $8.038 \mathrm{e}+026$ & -5 & 2000 \\
\hline
\end{tabular}

\section{CONCLUSION AND DISCUSSION}

In this paper we introduce the new method of laser fusion for $D+{ }^{3} \mathrm{He}$ reaction by using pettawat laser and also we calculate and consider penetrability and formation factor of the cloud. Using the results calculated, we obviously see the dependence of formation factor of the cloud on the intensity of laser field applied. Also the nuclear potential including mesons. In center of the nucleus play an important role in hole- boring coulomb potential coulomb barrier that was not anticipated before. Among these subjects, penetrability mainly increases. As shown in Fig. 1 and Fig. 2 and results from Table I we see an important increase in laser power around the region of $10^{24}$ to $10^{26}\left(\frac{\mathrm{W}}{\mathrm{cm}^{2}}\right)$ in comparison with quantum- well model that is because of the effect of nuclear potential surround the center. As show in Fig. 3 and Fig. 4 and regarding the numerical results in Table II we see that the value of the formation factor of the cloud strongly depends on the number of cycles, energy and the strength of laser field applied, In this new scheme for enough penetrability in order to hole- boring we need a laser with a power of more than 100 pw. But, since ignition is not necessary. So we don't need the symmetry and uniformity of lasers. In this paper by considering $\mathrm{D}+{ }^{3} \mathrm{He}$ reaction we have access to clean energy. Reducing in expenses in order to reach accessible efficiency is because of hole- boring and formation of cloud and increasing the rate of fusion reaction.

\section{REFERENCES}

[1] K. S, Krane, "Introductory Nuclear Physics," 1st Edition, John Wiley and Sons Ltd., vol. 35, 1988.

[2] A. Balantekin and N. Takigawa, "Quantum tunneling in Nuclear fusion," Rev.Mod.Phys., vol. 70, 1998, pp. 77-100.

[3] J. Nuckolls, L. Wood, A. Thiessen, and G. Zimmerman, "Laser compression of matter to super- high density," Nature, vol. 239, 1972, pp. 139-143.

[4] K. Imasaki and D. Li, "An approach of laser induced nuclear fusion," Laser Part, vol. 26, 2008, pp. 3-7.

[5] J. Lilley, "Nuclear Physics," NewYork, USA: John Wlley \& Sons, 2001.

[6] K. Imasaki and D. Li, "New Laser Fusion and Its Gain by Intense Laser," Energies, vol. 3, 2010, pp. 1176-1193.

[7] L. J. Wittenberg et al., "A Review of Helium-3 Resources and Acquisition for Use as Fusion Fuel," Fusion Technologhy, vol. 21, 1992 , p. 2230.

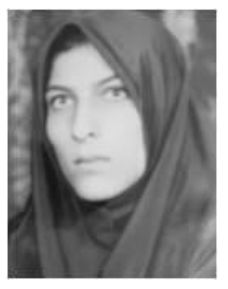

Seyeddeh Nasrin Hosseinimotlagh received her Ph.D and MS degrees from the Physics Department of Shiraz University in Iran.

She is an assistant professor in Department of Physics, Shiraz branch Islamic Azad University, Shiraz, Iran.. She has published in various journals. Department of Physics,Sciences College,Shiraz branch Islamic Azad University, Pardis,Sadra town, Shiraz, Iran.



Sara Sadeghi received her B.S from Department of Physics, Shiraz branch Islamic Azad University, Shiraz, Iran. She is university student in M.S in Atomic molecular physics. She has published in various journals Department of Physics,Sciences College,Shiraz branch Islamic Azad University, Pardis,Sadra town, Shiraz, Iran.

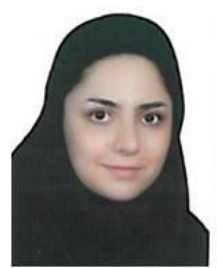

Fatemeh Mozafarikoroosh received her B.S from Department of Physics, Shiraz branch Islamic Azad University, Shiraz, Iran. She is university student in M.S in Dense Material Physics. She has published in various journals. Pardis Islamic Azad University of Shiraz, Department of Physics, Sciences College, Shiraz branch Islamic Azad University, Pardis, Sadra town, Shiraz, Iran 